\title{
Thanks to Reviewers
}

\author{
William M. Tierney ${ }^{7}$ and Martha S. Gerrity ${ }^{2}$ \\ ${ }^{1}$ Indianapolis, IN, USA; ${ }^{2}$ Portland VA Medical Center, P3MED, Portland, OR, USA.
}

$\mathrm{J}$ Gen Intern Med 23(12):2145-54

DOI: $10.1007 / \mathrm{s} 11606-008-0828-1$

(c) Society of General Internal Medicine 2008

$\mathrm{T}$ he quality of the papers published in the Journal depends on both the authors and the external reviewers who help the editors select the best papers and improve their presentation. JGIM is incredibly lucky to have such a wonderful group of reviewers: more than 1,100 individuals provided almost 1,500 reviews in the past year, with an average quality score of 4.3 on a 6-point Likert scale. (And our Deputy Editors are tough scorers!) Names of those who have reviewed for us from July 2007 through June 2008 are listed below. We hope this recognition will serve as a token of our gratitude. - The Editors

Eva Aagaard

J. Haxby Abbott

Jennifer Adams

Patience Agborbesong

Aref Agheli

Sacha Agrawal

Patricia Agre

Marilyn Aguirre-Molina

Elie Akl

Oluranti Aladesanmi

Amy Alderman

G. Caleb Alexander

Shelley Alexander

Irene Alexandraki

David Alfandre

Daniel Alford

Susan Allen

Dawn Alley

Wilma Alvarado-Little

Hugo Alvarez

Muhammad Amer

Robert Anda

Ruric (Andy) Anderson

Kathryn Anderson

Ida Androwich

Ashish Aneja

Dennis Ang

Jelili Apalara

Catherine Apaloo

Nedal Arar

Thurayya Arayssi

Katrina Armstrong

Scott Arnold

Sandra Arnold

Published online November 7, 2008
Vineet Arora

Melanie Arthur

John Astin

Brad Astor

Dexter Asuncion

Ayse Atasoylu

Steven Atlas

KoKo Aung

Moises Auron-Gomez

Ellen Averett

Brian Ayotte

Fatema Azam

Stewart Babbott

Sara Bachman

Jim Bader

Mark Baerlocher

Amy Baernstein

F. Amos Bailey

Jennifer Bailit

Matthew Bair

Tamilyn Bakas

David Baker

Suzanne Bakken

Tom Balcezak

Rajesh Balkrishnan

James Banks

MJ Barchman

David Bard

Aditya Bardia

Eve Bargmann

Judith C. Barker

randy barker

Gary Barnas

Jamie Barner

William Barnhart

Brendan Barrett

Paul Barrett

Michael Barry

Jeffrey Barsuk

Pat Bass

Sameh Basta

Carol Bates

Heidi Bauer

Carol Bayley

Janette Beals

Dilip Bearelly

Mary Beattie

Michael Beck

Edmund Becker

William Becker

Shawn Bediako

Cristina Belizna 
Kristin Bell

Douglas Bell

Katy Benjamin

Ian Bennett

Katherine Bensching

Loren Berenbom

Jessica Berg

Amy Bernstein

Lisa Bernstein

Dennis Bertram

Juanita Bezuidenhout

Surya Bhatt

Jay Bhattacharya

Janet Bickel

Beth Bierer

Andres Blei

Joan Blondin

Amy Blue

Ravi Bobba

Thomas Bodenheimer

Barbara Bokhour

Edward Bollard

Soo Borson

John Michael Bostwick

L. Ebony Boulware

Carol Bova

Patrick Bovier

Cynthia Boyd

Diane Boyle

Raymond Boyle

Thomas Brandon

Dale Bratzler

Ira Breite

Leslea Brickner

Patrick Bridge

Cedric Bright

Diane Brockmeyer

Laura Brockway

Frances Brokaw

Mark Bromberg

Joyce Bromberger

Robert Brooks

Blair Brooks

Daniel J. Brotman

David Brousseau

Arleen Brown

Rochelle Brown

William Browne

Chris Bryson

Peter Budett

Daniel Budnitz

Thuy Bui

Diana Buist

John Bulger

Barry Bunting

Marisha Burden

Alfred Burger

James Burgess

James Burks

Cynthia Burns

Lawton Burns

Harold Bursztajn
Susan Busch

Jada Bussey-Jones

J. Butler

Robert Byrd

James Byrd

Margaret Byrne

Christopher Callahan

Leigh Callahan

Wayne Campbell

Noll Campbell

Christopher Cannon

Peter H. Canter

John Canto

Kathleen Carey

Chris Carlucci

Patricia Carney

William Carpenter

James Carroll

Kimbroe Carter

Rachel Caskey

Kelly Caverzagie

Danelle Cayea

David Cella

Robert Centor

Isaac Cha

Chayan Chakraborti

Subani Chandra

Abhinav Chandra

Saurav Chatterjee

Sarwat Chaudhry

Ramachandra Rahul Chemitiganti

Daniel Chen

John Chen

Susan Cheng

Pramil Cheriyath

Michael Chernew

Barbara Chesney

John Chessare

Chi Chiao

Alyna Chien

Catherine Chittleborough

Joshua Chodosh

John Choe

John Christensen

Michael Christian

Cynthia Chuang

Nancy Claiborne

Jeanne Clark

Dan Clark

Sarah Clark

Roberta Clarke

J. Quentin Clemens

Nan Cochran

Rhonda Cockerill

Ellen Cohen

Steven Cohn

Cristin Colford

Joseph Collins

E. Dale Collins

Cathleen Colon-Emeric

John Concato

Michele Conde 
Douglas Conner

Rena Conti

Richard Conviser

Benjamin Cook

Cynthia Loveland Cook

Molly Cooke

Dagan Coppock

Eugene Corbett, Jr.

Kristina Cordasco

Leon Cosler

Steven Coughlin

Jack Coulehan

Ian Coulter

Michael Counte

Judith Covey

Patricia Cowper

Peter Cram

Elizabeth Crane

Fay Crawford

Ronald Crock

Peter Cronholm

Richard Cruess

Sylvia Cruess

Russell Cucina

Larry Culpepper

Megan Cunnane

Chinazo Cunningham

Farr Curlin

J. Randall Curtis

Jeffrey Curtis

Lowell Dale

Peter Danyi

Sumanth Daram

Jacqueline Darcey

Debasish Dasgupta

David Dausey

Michele David

Roger Davis

Neal Dawson

Hollis Day

Carolyn De Coster

Thomas De Fer

Mary de Groot

Pascal de Groote

$\mathrm{R}$ de Haan

Raymond De Vries

Raisa Deber

Mark Deeg

Kristy Deep

Tom Delbanco

Anne Dembitzer

Gerald Denton

Eric Deppert

Allan Detsky

Anandan Devarajan

Michelle Devor

Darren DeWalt

Dawn DeWitt

Paul Dexter

Richard Deyo

Kent DeZee

James Diamond
Aashish Didwania

Donna Diers

Diane Dietzen

Lisa Dixon

Estrelita Dixon

Alexander Djuricich

Steven Dobscha

Michael Donahoe

Karen Donelan

Ronald Dorotka

Emanuel Doyne

Benjamin Druss

Sascha Dublin

F. Daniel Duffy

David Dugdale

Daniel Dunham

Robert Dunlay

Patrick Dunn

Denise Dupras

Raegan Durant

Steven Durning

Liselotte Dyrbye

Kerry Eby

David Edelman

Mark Edlund

Brad Edwards

Erin Egan

Maniza Ehtesham

Jon Eisenhandler

David Eisenman

Eric Eisenstein

Arnold R. Eiser

Nancy Elder

Jon Elhai

Anne Elixhauser

Joann Elmore

Michael Elnicki

Andrea Ely

Peter Embi

Honora Englander

Adrian Espinosa

Marie-Louise Essink-Bot

Kevin Eva

Daniel Evans

George Everett

Ngozi Ogbunamiri Ezike

Anne Fabiny

Angela Fagerlin

Kathleen Fairfield

Ruth Falik

Donna Farley

Jeanne Farnan

Stephanie Faubion

Siri Fauli

Douglas Federman

Alicia Fernandez

Leonor Fernandez

Donna Fick

David Fiellin

Gerda Fillenbaum

Michael Fingerhood

David Fitzmaurice 
Lee Fleisher

Steven Fleming

John Fontanesi

John Fortney

Chris Fox

Michael Fox

Mony Fraer

Joshua Freeman

Allen Fremont

Kevin Frick

Terri Fried

Mark Friedberg

Erica Friedman

Harley Friedman

Susan Friedman

John Frohna

Alice Frohna

Dominic Frosch

Floyd Frost

Adriane Fugh-Berman

Michael Fuller

Vicki Fung

Angelo Gaffo

Diane Galbraith RN

Michael Galindo

Francesca Gany

Michael Ganz

Linda Ganzini

Raul Garcia

Reed M. Gardner

Carol Brooks Gardner

Brian Garibaldi

G. Waldon Garriss, III

Darrell Gaskin

Kumar Gaurav

Daniel Gaylin

Berta Geller

Susan George

Cynthia Geppert

Estella Geraghty

Megan Gerber

Dimple Ghassi

Todd Gilmer

Aaron Gilson

Peter Glassman

Marshall Glesby

Marcy Glisczinski

Lise Lotte Gluud

John Goddeeris

Zachary Goldberger

Nora Goldschlager

Jennifer Goldstein

Rachel Goldstone

Allen Goodman

John Goodson

Susan Goold

Howard Gordon

Ved Gossain

Michael Gould

Marc Gourevitch

Mark Graber

Christine Grady
Richard Grant

Michael Grasso

Martha Grayson

Marianne Green

Deborah Greenberg

David Greenburg

Jessica Gregg

Tim Grennan

Michael Grey

Joan Griffin

Marie Griffin

Michael Grodin

Susan Grossman

Ann Gruber-Baldini

Moti Grupper

Gretchen Guiton

Jyoti Gulia

Betsy Gunnels

Rahul Gupta

Sameer Gupta

Ramiro Gutierrez

Mary Haan

Kristin Hahn-Cover

Mark Hall

Judith Hall

Kirsten Hall Long

David Halle

Rachel Halpern

Andrew Hamilton

Jini Han

Elizabeth Haney

Kathleen Hanley

Charin Hanlon

Jennifer Hanrahan

Janice Hanson

Brenda Hanson

Barbara Hanusa

Laura Hanyok

Joseph Hardman

Jeffrey Harman

Rita Harmata

Heather Harrell

Kenneth Harris

Rebecca Harrison

Jason Hartig

Kelly Haskard

Mohamad Hatahet

Rose Hatala

David Hatem

Karen Hauer

Joshua Hauser

Sarah Hawley

Patricia Hayes

Rodney Hayward

Anna Headly

Paul Hebert

Frederick Hecht

Ira Helenius

Fred Hellinger

Paul Hemmer

Sean Hennessy

Carsten Hering Nielsen 
Caridad Hernandez

Charles Hershey

Rachel Hess

LeRoi Hicks

Lanis Hicks

Esther Hing

Susan Hingle

Katherine Hochman

Andrew Hoellein

Eric Holmboe

Margaret Holmes

Robert Holmes

Margaret Holmes-Rovner

Margaret Horlick

Susan Horn

Carol Horowitz

Leora Horwitz

Thomas Houston

Virginia Howard

Grace Huang

Elbert Huang

Thomas Huddle

Teresa Hudson

Mark Hughes

Chanita Hughes-Halbert

Alan Hunter

Jessica Hwang

Linda Hynan

Sanae Inagami

Orna Intrator

Thomas Inui

Catherine Ipsen

Matilde Irigoyen

Madelyn Iris

Amid Ismail

S. Barry Issenberg

Archana Jain

Gaurav Jain

Robert Jameson

Robert Jamison

Dorte Jarbol

Melanie Jay

Ian Jennings

Russell Joffe

Kirsten Johansen

Bruce Johnson

Julie Johnson

Kimberly Johnson

Timothy Johnson

Jeffrey Johnson

William Johnson

Therese Jones

Richard Jones

Katherine Jones

Cynthia Jorgensen

Alan Jotkowitz

Geoffrey Joyce

Amy Justice

Michael D. Justiss

Scott Kaatz

David Kagen

Mari Kai
Seth Kalichman

Lynne Kallenbach

Joel Kallich

Mohammad Kanjwal

Balavenkatesh Kanna

Lawrence Kaplan

Lori Karan

Leah Karliner

Cary Kart

Andrew Karter

Wesley Kasprow

Wayne Katon

Cornelius Katona

Joel Katz

Mitchell Katz

Jack Kaufman

Himangi Kaushal

Nancy Keating

Craig Keenan

San Keller

Amy Kelley

Ann Kenny

Lisa Keranen

Eve Kerr

Stefan Kertesz

Diana Kerwin

Patricia Ketsche

Natalie Key

Amor Khachemoune

Mutahir Khan

Arif Khan

Babar Khan

Rashid Khan

Aman Khurana

Bryce Kiberd

Christina Kile

Charles Kilo

Scott Kim

John Kimberly

Gerrit K. Kimsma

Danielle King

Lisa Kirkland

Weekitt Kittisupamongkol

Jack Kleinman

James Kleshinski

Mary Klingensmith

Sara Knight

Chris Knight

Merril Knudtson

Jeff Kohlwes

Shunzo Koizumi

Joseph Kolars

Anna Kolpakchi

Deborah Korenstein

Lynn Kosowicz

Michel Kossovsky

Christine Kovner

Anthony Kovner

Steven Kravet

Erin Krebs

Nancy Kressin

Selim Krim 
Robert Krippendorf

Ranjani Krishnan

Jennie Jacobs- Kronenfeld

Ian Kronish

Andrea Kronman

David Krummen

Edward Krupat

Ian Kudel

Satyarth Kulshrestha

Gautam Kumar

Hillary Kunins

Robert F. Kushner

Rene Lafreniere

Cindy Lai

Christine Laine

David Lairson

Geoffrey Lamb

David Latini

Judith Lave

Val Lawrence

Cathy Lazarus

Shoou-Yih Lee

Todd Lee

Robert Lee

H. Stephen Leff

Joseph LeMaster

Blake Lesselroth

Daniel Lessler

Sharon Leung

James Levenson

Peter Levin

Jeffrey Levin-Scherz

Cari Levy

Joel Lexchin

Alexander Li

Peter Lichstein

Amy Lief

Chyongchiou Lin

Jenny Lin

Jeffrey Linder

Zhimei Liu

Jeremy Long

Beaufort Longest

Lenny Lopez

John Lowe

Julie Lowery

Lee $\mathrm{Lu}$

Gregory Lucas

George Ludgate

Anna Lukowski

Jon Lurie

Nicole Lurie

May Nawal Lutfiyya

Frances Lynch

Monica Lypson

Matthew Maciejewski

Michael Maciosek

Palmer MacKie

Theodore MacKinney

Elizabeth Madigan

Mario Madruga

Pamela Anderson- Mahoney
Eldon Mahoney

Lois Maiman

Sumit Majumdar

Tariq Malik

C. Kevin Malotte

Nora Maloy

Ishak Mansi

Wickliffe Many, Jr.

Douglas Mapel

Erin Marcus

Melissa Margolis

Jennifer Mariotti

Elizabeth Markson

Leona Markson

Thomas Marrie

Michelle Martin

Richard Martin

Michelle Martin

Rajeev Mathavan

Dan Matlock

Patrick Mauldin

Charles Maynard

Lindsay Mazotti

Dennis Mazur

David McCaffrey

Jeffery McCombs

William McGaghie

Thomas McGinn

Margaret McLaughlin

Michelle McMacken

Melissa McNeil

Barbara Melamed

Michelle Mello

Jennifer Mellor

Tanya Melnik

David Meltzer

Geraldine Menard

Terrie Mendelson

Stephen Mennemeyer

Lisa Meredith

Elizabeth Merrick

Mark Meterko

Brian Meyer

James Michelson

Frank Michota

Chad Miller

Redonda Miller

Paul Miller

Christopher Millett

Paul Milligan

Farida Millwala

Sandra Mini

Matthew Mintz

Meena Mital

Sumit Mohan

JC Mohr

Elizabeth Momany

Patrick Monahan

Delfi Mondragon

Isaac Montoya

Sandra Moody-Ayers

Miriam Morey 
Keith Morgenlander

Hal Morgenstern

Laura Morrison

Jana Mossey

David Mount

Zachary Mulkey

Marlon Mundt

Andres Munoz

Harvey Murff

Rachel Murkofsky

Elizabeth Murray

Kyle Muus

Evan Myers

Janet Myers

Jennifer Myers

Jagdish Nachnani

Mohan Nadkarni

Joel Napenas

Robert Nardino

Masahiro Narita

David Nash

Trenton Nauser

J. Matthew Neal

David Neely

Robert Neilson

Karin Nelson

Mathieu Nendaz

Joan Neuner

Robert Newcomer

Anne Newland

Stephen Newman

Quyen Ngo-Metzger

Tung Nguyen

Giang Nguyen

Satyen Nichani

Christina Nicolaidis

Craig Nielsen

Gordon Noel

Saira Noor

John Norcini

Geoff Norman

Paul Nutting

Joseph ODonnell

John O'Donnell

Gbenga Ogedegbe

Greg Ogrinc

Gwendolyn O'Keefe

Janet O'Keeffe

Kevin O'Leary

Merrikay Oleen-Burkey

Mark Olfson

Patricia O'Sullivan

Renee Otter

Pamela Owens

Mary Pak

Carlos Palacio

Louis Pangaro

Nikolaos Papanas

Panos Papanikolaou

Ashish Parikh

Sharon Parish

Malcolm Parker
Patrician Parkerton

Mital Patel

Debora Paterniti

Michael Patterson

Valory Pavlik

Ora Pearlstein

Richard Pels

Jose A. Perez Jr.

Jonathan Perlin

Stephen Persell

Bernice Pescosolido

Anne Peters

Hoangmai Pham

Russell Phillips

Michael Phy

A. Simon Pickard

Carl Pieper

Harold Pincus

Roshini Pinto-Powell

Steven Pizer

Michael Polen

Craig Pollack

Harold Pollack

Charlene Pope

Arnold Potosky

Frank Potter

Nadereh Pourat

Isaac Powell

Benjamin Powers

Irene Prabhu Das

Eboni Price

David Price

Judith Prochaska

Peter Pronovost

Jacqueline Pugh

Elaine Puleo

Joseph Rabatin

Howard Rabinowitz

Judith Rabkin

Tiffany Radcliff

Paul Radensky

Susan Radius

Michael Radzienda

Vijay Rajput

Subha Ramani

Joe Ramsdell

Sumant Ranji

Karen Rascati

Neda Ratanawongsa

Susan Rawl

Susan Reed

Douglas Reifler

Joseph Rencic

Allen Repp

James Reschovsky

Thomas Ricketts

Janet Riddle

William Rifkin

Sally Rigler

Gerald Riley

Doron Rimar

Alfred Rimm 
David Rind

Cathy Risdon

Nicole Roberts

Douglas Roblin

Todd Rockwood

Richard Roetzheim

Theresa Rohr-Kirchgraber

Matthew Rondina

Michael Rosenblum

Robert Rosenheck

Gary Rosenthal

Ann-Marie Rosland

Stephen Ross

Rita Rossi-Foulkes

Michael Rotblatt

Craig Roth

Marilyn Rothert

Russell Rothman

Christianne Roumie

Michael Roy

Jorge Ruiz

Robin Ruthazer

George Rutherford

Carolyn Rutter

Kathryn Ryder

Kenneth Saag

Dana Safran

Amanda Salanitro

Talya Salant

Shadi Saleh

Ralph Salvietti

Jeanne Salyer

Jeffrey Samet

Lewis Sandy

Kiran Sarikonda

Franco Sassi

Elizabeth Sastre

Robert Saywell, Jr.

Thomas Scalea

Marilyn Schapira

Gordon Schectman

Joel Schectman

Martin Scherer

Sheira Schlair

Steven Schmidt

John Schmidt

Julie Schmittdiel

Michelle Schneidermann

Robert Schnoll

Herbert Schulberg

Henry Schultz

Richard Schulz

Mark Schwartz

Chris Sciamanna

Blanca Sckell

Don Scott

Alicia Scott-Wright

James Sebastian

Raymond CS Seet

Susan Sefcik

Joe Selby

Thomas Selden
Hilary Seligman

Janet Senf

Laura Sessums

Arjinder Sethi

Sanjay Sethi

Hiren Shah

Nirav R. Shah

Terry Shaneyfelt

David Shapiro

Johanna Shapiro

Lisa Sharp

Dennis Shea

Abdullah Shehab

Paul Shekelle

Robert Sheldon

Jay Shen

Changyu Shen

Qing Shen

Karen Sherman

Brad Sherman

Amy Ship

Michael Shlipak

Amir Shmueli

Ronald Shorr

William Shrank

Lynne Shuster

David Siegel

Scot Silverstein

David Simel

Todd Simon

Linas Simonaitis

Daniel Singer

Siddhartha Singh

Nishith K Singh

Rita Singhal

Kaycee Sink

Christine Sinsky

Deborah Sleight

Philip Sloane

Stephen Small

M.E. Beth Smith

Peter Smith

Alexander Smith

Kimberly Smith

Robert Smith

John Snyder

Dejano Sobral

Muhammad Sohail

Mildred Solomon

Ali Sonel

Seema Sonnad

Amit Sood

Paul Sorum

John Spangler

Claire Spettel

Joan St. Onge

Rachel Stark

Helene Starks

Joanna Starrels

Terrence Steyer

Margaret Stineman

Steven Stoltz 
Sheryl Strasser

Jennifer Strauss

Brian Strom

Ira Strumwasser

Bruce Stuart

George Stukenborg

Joachim Sturmberg

Tze-Jung Su

Jeremy Sugarman

Andrew Sumner

Walton Sumner

Jian Sun

Sung Wu Sun

Sanjeev Suri

Paul Sutton

Marika Suttorp

Karen Swanson

Karen Szauter

Andrew Talal

Zohray Talib

Stephen Taplin

Derjung Tarn

Christine Taylor

Ildefonso Tellez

Sara Templin

Eric Terman

Kevin Terrell

Norma Terrin

Thenappan Thenappan

Kris Thomas

Cindy Parks Thomas

Stephanie Thompson

Carolyn Thorpe

Jon Tilburt

John Tilford

Wolfgang Tillinger

Imad Tleyjeh

Gregory Todd

Anthony Tommasello

Alexia Torke

John Townes

Bruce Trigg

David Trochtenberg

Robert Trowbridge

Paula Trzepacz

Apostolos Tsapas

Chien-Wen Tseng

James Tulsky

Laurene Tumiel-Berhalter

Alexander Turchin

Roxanne Tyroch

George Tzanis

Toshiko Uchida

Dan Ullmann

Steven Ullmann

Carole Upshur

Patrice A. Caetano Vaeth

Prashant Vaishnava

Vivian Valdmanis

Marcia Valenstein

Terence Valenzuela

Jerome Van Ruiswyk
Jonathan VanGeest

Orly Vardeny

Eduard Vasilevskis

Celso Velazquez

Vicente Jose Velez

Jon Veloski

Craig Velozo

John Vetto

Heimo Viinamaki

Rey Vivo

Angelo Volandes

Robert Volk

Joanna Volpe-Vartanian

Keith vom Eigen

Julie Wagner

Terry Wahls

Dietlind Wahner-Roedler

Timothy Waidmann

Louise Walter

George Wang

Hha Wang

JianLi Wang

Lawrence Ward

Molly Waring

Donna Washington

Peter Watson

Alice Watson

Delese Wear

Christina Wee

Edgard Wehbe

Richard Weinberg

Saul Weiner

Robin Weinick

Ruth Weinstock

Sheila Weiss

Maria Jibaja Weiss

Martin Were

Albert Wertheimer

Mark Wess

Colin West

Terrie Wetle

Scott Wetterhall

Margaret Wheeler

Heather Whelan

Richard White

Kelly White

Susan Whitley

Jeffrey Wiese

Consuelo Wilkins

Donna Williams

Amy Wilson

Lucy Wilson

Ira Wilson

Donna Windish

Nancy Winslade

Christopher Wittich

Marshall Wolf

Leah Wolfe

Fredric Wolinsky

Roger Wong

Mitchell Wong

Raymond Wong 
Victoria Wong

Sabrina Wong

Judith Wooldridge

Scott Wright

Adam Wright

Curtis Wright

Jeffrey Wright

Richard Wyderski

Leon Wyszewianski

Bing Xue

William Yancy, Jr.

Yi Yang

Ellen Yee

Balaji Yegneswaran

Joyce Yeh

Michael Yi

Constantin Yiannoutsos
Hsiang Yin

Ernest Yoder

Marianne Ulcickas Yood

Angela York

William Yost

Joyce You

Sharon Youmans

Fred Zar

Alan Zaslavsky

John Zeber

Philip Zeitler

Shannon Zenk

Brian Zikmund-Fisher

Corresponding Author: William M. Tierney; 410 West Tenth Street Suite 2000,Indianapolis, IN 46202, USA (e-mail: wtierney@iupui.edu). 